\title{
Cisplatin and fluorouracil with or without panitumumab in patients with recurrent or metastatic squamous-cell carcinoma of the head and neck (SPECTRUM): an open-label phase 3 randomised trial
}

\author{
Jan B Vermorken, Jan Stöhlmacher-Williams, Irina Davidenko, Lisa Licitra, Eric Winquist, Cristian Villanueva, Paolo Foa, Sylvie Rottey, \\ Krzysztof Skladowski, Makoto Tahara, Vasant R Pai, Sandrine Faivre, Cesar R Blajman, Arlene A Forastiere, Brian N Stein, Kelly S Oliner, \\ Zhiying Pan, Bruce A Bach, on behalf of the SPECTRUM investigators
}

\section{Summary}

Background Previous trials have shown that anti-EGFR monoclonal antibodies can improve clinical outcomes of patients with recurrent or metastatic squamous-cell carcinoma of the head and neck (SCCHN). We assessed the efficacy and safety of panitumumab combined with cisplatin and fluorouracil as first-line treatment for these patients.

Methods This open-label phase 3 randomised trial was done at 126 sites in 26 countries. Eligible patients were aged at least 18 years; had histologically or cytologically confirmed SCCHN; had distant metastatic or locoregionally recurrent disease, or both, that was deemed to be incurable by surgery or radiotherapy; had an Eastern Cooperative Oncology Group performance status of 1 or less; and had adequate haematological, renal, hepatic, and cardiac function. Patients were randomly assigned according to a computer-generated randomisation sequence (1:1; stratified by previous treatment, primary tumour site, and performance status) to one of two groups. Patients in both groups received up to six 3-week cycles of intravenous cisplatin $\left(100 \mathrm{mg} / \mathrm{m}^{2}\right.$ on day 1 of each cycle) and fluorouracil $\left(1000 \mathrm{mg} / \mathrm{m}^{2}\right.$ on days $1-4$ of each cycle); those in the experimental group also received intravenous panitumumab ( $9 \mathrm{mg} / \mathrm{kg}$ on day 1 of each cycle). Patients in the experimental group could choose to continue maintenance panitumumab every 3 weeks. The primary endpoint was overall survival and was analysed by intention to treat. In a prospectively defined retrospective analysis, we assessed tumour human papillomavirus (HPV) status as a potential predictive biomarker of outcomes with a validated p16-INK4A (henceforth, p16) immunohistochemical assay. Patients and investigators were aware of group assignment; study statisticians were masked until primary analysis; and the central laboratory assessing p16 status was masked to identification of patients and treatment. This trial is registered with ClinicalTrials.gov, number NCT00460265.

Findings Between May 15, 2007, and March 10, 2009, we randomly assigned 657 patients: 327 to the panitumumab group and 330 to the control group. Median overall survival was $11 \cdot 1$ months $(95 \% \mathrm{CI} 9 \cdot 8-12 \cdot 2)$ in the panitumumab group and $9 \cdot 0$ months $(8 \cdot 1-11 \cdot 2)$ in the control group (hazard ratio [HR] 0 873, 95\% CI 0 729-1.046; p=0 1403). Median progression-free survival was 5.8 months (95\% CI 5.6-6.6) in the panitumumab group and 4.6 months $(4 \cdot 1-5 \cdot 4)$ in the control group (HR $0 \cdot 780,95 \%$ CI $0 \cdot 659-0 \cdot 922 ; p=0 \cdot 0036)$. Several grade 3 or 4 adverse events were more frequent in the panitumumab group than in the control group: skin or eye toxicity $(62$ [19\%] of 325 included in safety analyses vs six [2\%] of 325), diarrhoea (15 [5\%] vs four [1\%]), hypomagnesaemia (40 [12\%] vs 12 [4\%]), hypokalaemia (33 [10\%] vs 23 [7\%]), and dehydration (16 [5\%] vs seven [2\%]). Treatment-related deaths occurred in 14 patients $(4 \%)$ in the panitumumab group and eight $(2 \%)$ in the control group. Five $(2 \%)$ of the fatal adverse events in the panitumumab group were attributed to the experimental agent. We had appropriate samples to assess p16 status for 443 (67\%) patients, of whom $99(22 \%)$ were p16 positive. Median overall survival in patients with p16-negative tumours was longer in the panitumumab group than in the control group $(11.7 \mathrm{months}$ [95\% CI 9.7-13.7] vs $8 \cdot 6$ months [6.9-11.1]; HR 0.73 [95\% CI 0.58-0.93]; $\mathrm{p}=0.0115)$, but this difference was not shown for p16-positive patients $(11 \cdot 0$ months [7.3-12.9] vs $12 \cdot 6$ months [7.7-17.4]; 1.00 [0-62-1.61]; p=0 998). In the control group, p16-positive patients had numerically, but not statistically, longer overall survival than did p16-negative patients (HR $0 \cdot 70$ [95\% CI 0.47-1·04]).

Interpretation Although the addition of panitumumab to chemotherapy did not improve overall survival in an unselected population of patients with recurrent or metastatic SCCHN, it improved progression-free survival and had an acceptable toxicity profile. p16 status could be a prognostic and predictive marker in patients treated with panitumumab and chemotherapy. Prospective assessment will be necessary to validate our biomarker findings.

Funding Amgen Inc.

Lancet Oncol 2013; 14: 697-710 Published Online June 6, 2013 http://dx.doi.org/10.1016/ S1470-2045(13)70181-5

See Comment page 672 Antwerp University Hospital, Edegem, Belgium (J B Vermorken MD); Universitätsklinikum Carl Gustav Carus, Dresden, Germany (J Stöhlmacher-Williams MD); Krasnodar City Oncology Center, Krasnodar, Russia (I Davidenko MD); Fondazione IRCCS Istituto Nazionale Tumori, Milan, Italy (L Licitra MD); London Health Sciences Centre, London, ON, Canada (E Winquist MD); University Hospital of Besançon, Besançon, France (CVillanueva MD); San Paolo University Hospital, Milan, Italy (P Foa MD); Ghent University Hospital, Ghent, Belgium (S Rottey MD); Instytut im Marii Skłodowskiej-Curie, Gliwice, Poland (K Skladowski MD); National Cancer Center Hospital East, Kashiwa City, Japan (M Tahara MD); Tata Memorial Hospital, Mumbai, India (V R Pai MD); Hôpital Beaujon, Clichy, France (S Faivre MD); Isis Clinica Especializada, Santa Fe, Argentina (C R Blajman MD); The Johns Hopkins Hospital, Baltimore, MD, USA (A A Forastiere MD); Adelaide Cancer Centre, Kurralta Park, SA, Australia (B N Stein MBBS) and Amgen Inc, Thousand Oaks, CA, USA (K S Oliner PhD, Z Pan PhD, B A Bach MD)

Correspondence to: Dr Jan B Vermorken, Department of Medical Oncology, Antwerp University Hospital, Wilrijkstraat 10, 2650 Edegem, Belgium jan.b.vermorken@uza.be 


\section{Introduction}

Platinum-based combination chemotherapy regimens can be used to treat patients with incurable locoregionally recurrent or metastatic squamous-cell carcinoma of the head and neck (SCCHN). ${ }^{1}$ Although these regimens lead to tumour responses in about $10-35 \%$ of patients, median survival is less than 1 year and the effects on patients' quality of life are unknown..$^{2-6}$

Dysregulation of the EGFR signalling pathway plays a part in the development and progression of SCCHN., Clinical trials ${ }^{6,9}$ have shown that addition of anti-EGFR monoclonal antibodies to chemotherapy improves clinical outcomes in patients with recurrent or metastatic SCCHN. In a randomised phase 3 study in the USA, more patients in the group given cisplatin plus cetuximab responded than in the group receiving cisplatin plus placebo; and in a randomised phase 3 study (EXTREME) in Europe, ${ }^{9}$ addition of cetuximab to cisplatin and fluorouracil or to carboplatin and fluorouracil improved overall survival.

Human papillomavirus (HPV) DNA has been detected in up to two-thirds of oropharyngeal SCCHN tumours in patients presenting with locoregionally advanced disease. ${ }^{10,11}$ Patients with locoregionally advanced HPVpositive oropharyngeal SCCHN who are treated with radiotherapy with or without chemotherapy have a better outlook than do HPV-negative patients. ${ }^{11-16}$ However, the global prevalence and prognostic effect of $\mathrm{HPV}$ in patients with recurrent or metastatic SCCHN arising from the oropharynx, oral cavity, larynx, and hypopharynx is not well understood, particularly in the clinical trial setting. HPV-positive and HPV-negative SCCHN tumours differ in terms of biology, histology, genetic alterations, and prognosis..$^{11,13,17,18} \mathrm{HPV}$-positive SCCHN tumours are characterised by the presence of high-risk HPV DNA (most commonly HPV 16) and the coexpression of the viral oncoproteins E6 and E7, which modulate expression of key cellular proteins (such as the tumour suppressor p53 and retinoblastoma tumoursuppressor protein), leading to upregulated expression of the cyclin-dependent kinase inhibitor p16-INK4A (henceforth, p16). ${ }^{10,18,19}$ How HPV status affects the outlook of patients with recurrent or metastatic disease, or their response to treatment, is unknown. Some studies ${ }^{20-24}$ have suggested interactions between HPV and the EGFR signalling pathway. Therefore, HPV status (as assessed by p16 immunohistochemistry of formalinfixed paraffin-embedded samples) might affect outcomes during anti-EGFR treatment.

Panitumumab is a fully human anti-EGFR monoclonal antibody that is used both as a single agent and combined with chemotherapy for treatment of metastatic colorectal cancer. ${ }^{25}$ Preclinical data for SCCHN cell lines and xenografts showed more antitumour activity with panitumumab plus radiotherapy than with radiotherapy alone, ${ }^{26}$ and phase 1 response data for panitumumab plus chemoradiotherapy have suggested that additional investigation of panitumumab in SCCHN is warranted. ${ }^{27}$ In the Study of Panitumumab Efficacy in Patients With Recurrent and/or Metastatic Head and Neck Cancer (SPECTRUM), we compared panitumumab plus cisplatin and fluorouracil with chemotherapy alone as first-line treatment for recurrent or metastatic SCCHN. Additionally, we investigated the relative effect of treatment with panitumumab combined with chemotherapy in patients with recurrent or metastatic SCCHN who do (p16 positive) or do not (p16 negative) express p16.

\section{Methods \\ Study design and participants}

This open-label phase 3 randomised trial was done at 126 sites in 26 countries. Eligible patients were aged at least 18 years; had histologically or cytologically confirmed SCCHN; had distant metastatic or locoregionally recurrent disease, or both, that was deemed to be incurable by surgery or radiotherapy; had an Eastern Cooperative Oncology Group (ECOG) performance status of 1 or less; and had adequate haematological, renal, hepatic, and cardiac function. Patients who had received primary radiation therapy were eligible when locoregional recurrence was in the field of radiation and occurred at least 6 months after therapy completion, or when it was outside the field of radiation and occurred at least 3 months after therapy completion. Patients were excluded if they had received previous systemic chemotherapy for recurrent or metastatic SCCHN (unless part of multimodality treatment for locoregionally advanced SCCHN completed more than 6 months before study entry); had another primary cancer with treatment within 2 years of randomisation; had nasopharyngeal carcinoma or CNS metastases; had undergone major surgery in the previous 4 weeks or minor surgery in the previous 2 weeks; or had received previous anti-EGFR treatment (unless part of initial curative multimodality therapy).

The study protocol was approved by independent ethics committees at each participating centre. All participants provided written informed consent.

\section{Randomisation and masking}

Patients were randomly assigned (1:1) to one of two groups according to a computer-generated randomisation sequence (provided by an external vendor [ICON Clinical Research, North Wales, PA, USA]) with an automated interactive voice response system. Randomisation was stratified by previous treatment (newly diagnosed or previously untreated vs recurrent disease), primary tumour site (combined hypopharynx and oral cavity vs combined oropharynx and larynx), and ECOG performance status (0 vs 1). Double-blind treatment assignment was not possible because of the characteristic rash associated with EGFR inhibitors, including monoclonal antibodies such as panitumumab. ${ }^{28}$ Patients and investigators were aware of group assignment, and study statisticians were masked 
until the primary analysis. Pathologists doing p16 immunohistochemical assays were masked to patient identification and treatment.

\section{Procedures}

Patients in the panitumumab group received cisplatin and fluorouracil plus panitumumab, and those in the control group received cisplatin and fluorouracil alone. All patients received $100 \mathrm{mg} / \mathrm{m}^{2}$ intravenous cisplatin on day 1 of a 3-week cycle, and $1000 \mathrm{mg} / \mathrm{m}^{2}$ intravenous (continuous) fluorouracil infusion on days 1-4 of each cycle. Carboplatin (target area under the curve by the Calvert formula $5 \mathrm{mg} / \mathrm{mL}$ per min) could be permanently substituted for cisplatin when patients had creatinine clearance of less than $50 \mathrm{~mL} / \mathrm{min}$ or grade 2 neurological toxicity (eg, sensory or motor neuropathy and ototoxicity). Patients in the panitumumab group received $9 \mathrm{mg} / \mathrm{kg}$ intravenous panitumumab on day 1 of each cycle immediately before receiving chemotherapy.

Treatment continued until disease progression or for a maximum of six 3-week cycles. Patients who discontinued one study drug could receive the remaining drugs until completion of six cycles, disease progression, intolerable toxicity, or study withdrawal. Protocol-specified dose modifications and interruptions of study drugs were allowed when patients experienced toxicity (appendix). Patients in the panitumumab group who had not had disease progression after six cycles could choose to receive panitumumab until disease progression, intolerable toxicity, or study withdrawal.

Tumour response was assessed by CT or MRI at baseline and then every 6 weeks until disease progression. As per Response Evaluation Criteria in Solid Tumors (RECIST; version 1.0), ${ }^{29}$ complete or partial tumour responses were confirmed at least 4 weeks after the initial response assessment. Patients who discontinued treatment were followed up to obtain data for safety (30 days after the last treatment), survival (every 3 months until 3 years after the last patient underwent randomisation), and subsequent treatment for SCCHN (every 3 months until 3 years after the last patient underwent randomisation).

Adverse events occurring during the study were graded with the National Cancer Institute Common Terminology Criteria for Adverse Events (version 3.0). ${ }^{30}$ Clinical and laboratory assessments were done at screening, on day 1 of each cycle, and during safety follow-up. Serum samples for anti-panitumumab antibody analysis were obtained from patients in the panitumumab group before receiving infusions on day 1 of cycles 1 and 5 , every 6 months thereafter, and at the safety follow-up visit. The serum samples were analysed as described previously. ${ }^{31}$

Core biopsies $(1 \mathrm{~mm})$ from available formalin-fixed paraffin-embedded SCCHN tumour blocks (primary or metastatic sites) were obtained, sectioned, reviewed by a pathologist, and used to construct a tumour microarray.
In sites where blocks were not submitted, tissue slides were assessed. An immunohistochemical assay (p16INK4a Histology Kit, CINtec, Roche mtm laboratories AG, Heidelberg, Germany) that has been validated for testing of cervical cancer samples and qualified for assessment of $\mathrm{p} 16$ expression in SCCHN samples ${ }^{32}$ was used to determine tumour HPV status according to a prespecified plan. Tumour p16 expression was detected with the Clone E6H4 monoclonal antibody (Roche mtm laboratories AG, Heidelberg, Germany) and stained with a Nemesis Autostainer and diaminobenzidine secondary detection kit (Dako, Carpinteria, CA, USA). Samples were judged to be p16 positive when they had strong and diffuse nuclear and cytoplasmic staining in at least $10 \%$ of tumour cells; ${ }^{32}$ all other patients were defined as p16 negative. The immunohistochemical assay success rate was more than $99 \%$.

\section{Statistical analysis}

The primary endpoint was overall survival (time from randomisation to death); patients who had not died at the time of the primary analysis were censored on the date that they were last known to be alive. An estimated 470 deaths would provide $90 \%$ power to detect a hazard ratio (HR) of 0.7407 , with an $\alpha$ of 0.05 . With the assumptions that median overall survival in the control group would be 8.7 months (estimate based on assessment of clinical experience with platinum-based chemotherapy in recurrent or metastatic SCCHN) and an exponential distribution for overall survival, this HR would translate into a $35 \%$ relative and 3-month absolute

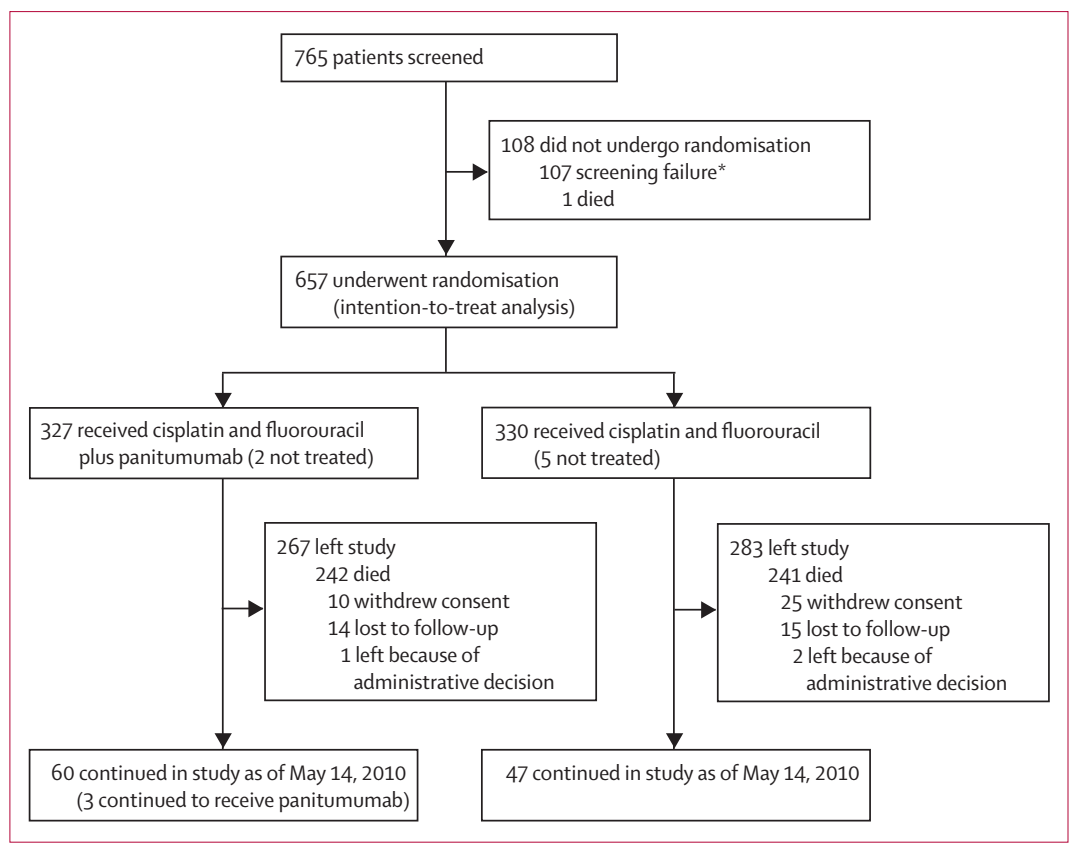

Figure 1: Trial profile

*34 did not meet disease-related eligibility criteria, 20 laboratory failure, 10 timing or toxic effects from previous treatment, 2 inadequate or inappropriate previous treatment, 1 withdrew consent, and 40 for other reasons. 
increase in median overall survival. We estimated that 650 patients would have to undergo randomisation in 20 months and have about 18 months of follow-up. The maximum planned study duration was 56 months.

Secondary endpoints were progression-free survival (PFS; time from randomisation to disease progression or death), proportions of patients who achieved an objective response (complete and partial responses combined),

\begin{tabular}{|c|c|c|}
\hline & Panitumumab group $(n=327)$ & Control group $(n=330)$ \\
\hline Men & $283(87 \%)$ & $287(87 \%)$ \\
\hline Women & $44(13 \%)$ & $43(13 \%)$ \\
\hline \multicolumn{3}{|l|}{ Geographical region } \\
\hline North America & $24(7 \%)$ & $25(8 \%)$ \\
\hline Western Europe & $101(31 \%)$ & $116(35 \%)$ \\
\hline Asia-Pacific & $54(17 \%)$ & $42(13 \%)$ \\
\hline South America & $25(8 \%)$ & $31(9 \%)$ \\
\hline Eastern Europe & $123(38 \%)$ & $116(35 \%)$ \\
\hline \multicolumn{3}{|l|}{ Ethnic origin } \\
\hline White & $268(82 \%)$ & $271(82 \%)$ \\
\hline Black & $4(1 \%)$ & $2(1 \%)$ \\
\hline Hispanic & $13(4 \%)$ & $12(4 \%)$ \\
\hline Asian & $25(8 \%)$ & $30(9 \%)$ \\
\hline Japanese & $13(4 \%)$ & $7(2 \%)$ \\
\hline Other* & $4(1 \%)$ & $8(2 \%)$ \\
\hline Age (years) & $58(53-63)$ & $59(53-64)$ \\
\hline$<65$ & $268(82 \%)$ & $256(78 \%)$ \\
\hline$\geq 65$ & $59(18 \%)$ & $74(22 \%)$ \\
\hline \multicolumn{3}{|c|}{ Eastern Cooperative Oncology Group performance status } \\
\hline 0 & $98(30 \%)$ & $98(30 \%)$ \\
\hline 1 & $227(69 \%)$ & $228(69 \%)$ \\
\hline $2 \dagger$ & $2(1 \%)$ & $4(1 \%)$ \\
\hline Duration of disease (months) & $15 \cdot 0(9 \cdot 6-26 \cdot 2)$ & $15 \cdot 9(10 \cdot 1-32 \cdot 5)$ \\
\hline \multicolumn{3}{|c|}{ Involuntary weight loss in the previous 6 months } \\
\hline $0-5 \%$ & $27(8 \%)$ & $28(8 \%)$ \\
\hline$>5 \%$ & $60(18 \%)$ & $68(21 \%)$ \\
\hline None or missing & $240(73 \%)$ & $234(71 \%)$ \\
\hline \multicolumn{3}{|l|}{ Primary tumour site } \\
\hline Oropharynx & $86(26 \%)$ & $96(29 \%)$ \\
\hline Hypopharynx & $52(16 \%)$ & $37(11 \%)$ \\
\hline Larynx & $100(31 \%)$ & 95 (29\%) \\
\hline Oral cavity & $89(27 \%)$ & $102(31 \%)$ \\
\hline \multicolumn{3}{|l|}{ Extent of disease } \\
\hline Locoregional recurrence only & $143(44 \%)$ & $126(38 \%)$ \\
\hline Distant metastatic & $119(36 \%)$ & $123(37 \%)$ \\
\hline $\begin{array}{l}\text { Distant metastatic and locoregionally } \\
\text { recurrent }\end{array}$ & $65(20 \%)$ & $81(25 \%)$ \\
\hline \multicolumn{3}{|l|}{ Primary tumour histological type } \\
\hline Well differentiated & $63(19 \%)$ & $63(19 \%)$ \\
\hline Moderately differentiated & $133(41 \%)$ & $123(37 \%)$ \\
\hline Poorly differentiated & $54(17 \%)$ & $70(21 \%)$ \\
\hline Undifferentiated & $6(2 \%)$ & $6(2 \%)$ \\
\hline \multirow[t]{2}{*}{ Not otherwise specified/unknown } & $71(22 \%)$ & $68(21 \%)$ \\
\hline & & (Continues on next page) \\
\hline
\end{tabular}

duration of response (DOR; time from first confirmed objective response to disease progression, per RECIST), time to response (TTR; time from randomisation to first confirmed objective response), and safety.

The primary analysis of overall survival and PFS was done by intention to treat. We assessed numbers of objective responses, TTR, and DOR in patients with at least one one-dimensionally measurable lesion at baseline, per RECIST. All randomly assigned patients who received at least one dose of panitumumab or chemotherapy were included in the safety analysis. An independent data monitoring committee did interim safety analyses on a roughly annual basis during the treatment phase.

We did between-group comparisons of overall survival and PFS with log-rank tests stratified by randomisation factors. When the difference in overall survival was not significant, all other $\mathrm{p}$ values were descriptive only. We estimated HRs and 95\% CIs for overall survival and PFS with a Cox model stratified by randomisation factors. We used the Cochran-Mantel-Haenszel method ${ }^{33}$ to assess the association between treatment group (panitumumab plus chemotherapy vs chemotherapy alone) and objective response (yes vs no), while controlling for randomisation stratification factors. We used the Wilson score method ${ }^{34}$ with continuity correction to calculate a $95 \%$ CI for the difference in numbers of objective responses. For patients with an objective response, we estimated DOR with the Kaplan-Meier method, and assessed TTR with descriptive statistics. We did post-hoc analyses to assess differences in adverse events between groups with Fisher's exact test, with no correction for multiple comparisons. Analyses were done in SAS (version 9.2).

We assessed all patients in the intention-to-treat dataset with formalin-fixed paraffin-embedded tumour samples for p16 status. We used data from the primary analysis in the retrospective analysis of overall survival and PFS by tumour p16 status. We used an unstratified univariate Cox model for comparisons of overall survival and PFS between p16 groups, and unstratified log-rank tests and Cox models to assess the treatment effect within p16 groups.

This study is registered with ClinicalTrials.gov, number NCT00460265.

\section{Role of the funding source}

The study sponsor was involved in study design, in collaboration with the corresponding author and members of the SPECTRUM steering committee; provision of access to the raw data to the study biostatistician (ZP); data management; prespecified statistical analyses; and data interpretation. JBV and BB led development of the report; all authors (some of whom are employed by the funder) participated in the writing of the report. The study sponsor funded medical writing assistance. The corresponding author had full access to 
all data in the study and had final responsibility for the decision to submit for publication.

\section{Results}

Between May 15, 2007, and March 10, 2009, we randomly assigned 657 patients to the two treatment groups (figure 1). Baseline characteristics were similar in the two groups (table 1). Seven patients did not receive treatment (figure 1); 325 patients in each group received at least one dose of panitumumab or chemotherapy and were included in safety analyses.

Of the 327 patients assigned to the panitumumab group, all discontinued chemotherapy (reasons given for discontinuation of each component; some patients counted more than once): 155 completed protocolspecified chemotherapy, 55 did not meet protocolspecified criteria $(29$ had creatinine clearance $<50 \mathrm{~mL} / \mathrm{min}$; 26 had grade 2 or 3 neurotoxicity), 46 had disease progression, 45 had an adverse event, 36 died, 31 requested discontinuation, eight withdrew consent, six were non-compliant, four were lost to follow-up, one was ineligible, and 15 for other reasons. 324 patients discontinued panitumumab at any point while in the study: 144 had disease progression, 48 requested discontinuation, 43 had an adverse event, 41 died, eight withdrew consent, seven were non-compliant, five were lost to follow-up, one was ineligible, and 27 for other reasons. Of the 330 assigned to the control group, all discontinued chemotherapy: 128 completed protocolspecified chemotherapy, 76 did not meet protocol specified criteria ( 55 had creatine clearance $<50 \mathrm{~mL} / \mathrm{min}$; 20 had grade 2 or 3 neurotoxicity; 1 had grade 4 neurotoxicity), 88 had disease progression, 47 had adverse event, 29 died, 16 requested discontinuation, 19 withdrew consent, five were non-compliant, two were lost to followup, one was ineligible, and 15 for other reasons.

At the time of the primary analysis on May 14, 2010, 550 patients had left the study: 483 had died, 35 had withdrawn their consent, 29 were lost to follow-up, and three left because of decisions made by the study oversight team. 153 patients in the panitumumab group and 147 patients in the control group received at least one subsequent treatment for SCCHN after discontinuation of protocol-specified treatment: 97 in the panitumumab group and 84 the control group received additional cytotoxic chemotherapy; 50 and 46 radiotherapy or surgery, or both; and 15 and 31 anti-EGFR monoclonal antibody therapy.

The median relative dose intensity of panitumumab during the study (proportion given of amount planned) was 91\% (IQR 83-98). The median number of 3-week cycles in which panitumumab was given was five (three to eight). The median duration of panitumumab treatment was 18 . 3 weeks $(9 \cdot 3-26 \cdot 9)$. At least one dose of panitumumab was withheld for 39 patients (12\%). 126 patients $(39 \%)$ in the panitumumab group were eligible to continue panitumumab after completing six cycles of chemotherapy

\begin{tabular}{|c|c|c|}
\hline & Panitumumab group ( $n=327)$ & Control group $(n=330)$ \\
\hline \multicolumn{3}{|l|}{ (Continued from previous page) } \\
\hline \multicolumn{3}{|l|}{ Previous treatment $\ddagger$} \\
\hline Chemotherapy or radiotherapy, or both & $267(82 \%)$ & $263(80 \%)$ \\
\hline \multicolumn{3}{|l|}{ Chemotherapy } \\
\hline Platinum & $128(39 \%)$ & $113(34 \%)$ \\
\hline Fluoropyrimidine & $52(16 \%)$ & $44(13 \%)$ \\
\hline Taxane & $7(2 \%)$ & $8(2 \%)$ \\
\hline Methotrexate & $9(3 \%)$ & $6(2 \%)$ \\
\hline Other & $15(5 \%)$ & $18(5 \%)$ \\
\hline Radiotherapy & $266(81 \%)$ & $259(78 \%)$ \\
\hline $\begin{array}{l}\text { Patients with locoregionally advanced } \\
\text { disease }\end{array}$ & $189(58 \%)$ & $187(57 \%)$ \\
\hline Surgery & $282(86 \%)$ & $284(86 \%)$ \\
\hline $\begin{array}{l}\text { Data are } \mathrm{n}(\%) \text { or median (range). }{ }^{*} \text { Australian } \mathrm{Ab} \\
\text { performance status of } 2 \text { were protocol violations } \\
\text { treatment in locoregionally advanced disease }>6\end{array}$ & $\begin{array}{l}\text { original, or unknown or missing. } \dagger \text { En } \\
\text { ₹Previous treatment given as adjuv } \\
\text { months before randomisation. }\end{array}$ & $\begin{array}{l}\text { olled patients with a } \\
\text { ant or part of multimodality }\end{array}$ \\
\hline
\end{tabular}

and panitumumab. 95 patients (75\% of those eligible; $29 \%$ of all patients in the panitumumab group) continued with panitumumab maintenance treatment, and went on to receive a median of three cycles (IQR two to eight) of panitumumab monotherapy. Panitumumab monotherapy varied by region (appendix).

The median relative dose intensity of cisplatin was $87 \%$ (IQR 75-97) in the panitumumab group and 85\% (72-98) in the control group, and the median relative dose intensity of carboplatin was 95\% (84-100) and 98\% (86-100). The median number of cisplatin cycles was $4 \cdot 0$ $(2 \cdot 0-6 \cdot 0)$ in the panitumumab group and $4 \cdot 0(2 \cdot 0-5 \cdot 0)$ in the control group. The median number of carboplatin cycles was $2 \cdot 0(2 \cdot 0-4 \cdot 0)$ in the panitumumab group and $4 \cdot 0(2 \cdot 0-5 \cdot 0)$ in the control group. The median duration of cisplatin treatment was $13 \cdot 4$ weeks $(7 \cdot 9-19 \cdot 3)$ in the panitumumab group and 13.0 weeks $(6 \cdot 4-18 \cdot 4)$ in the control group. Overall, 155 patients (24\%; 69 [21\%] in the panitumumab group; 86 [26\%] in the control group) switched from cisplatin to carboplatin. Fewer patients in the panitumumab group (29 [42\%]) than in the control group (52 [60\%]) switched because of a creatinine clearance of less than $50 \mathrm{~mL} / \mathrm{min}$. The median time to switching from cisplatin to carboplatin for any reason was 64 days (IQR 34-106) in the panitumumab group and 77 days (43-106) in the control group.

The median relative dose intensity of fluorouracil was $89 \%$ (79-97) in the panitumumab group and 90\% (79-99) in the control group. The median number of fluorouracil cycles was $5 \cdot 0(3 \cdot 0-6 \cdot 0)$ in the panitumumab group and $4.0(2 \cdot 0-6 \cdot 0)$ in the control group. The median duration of fluorouracil treatment was 17.9 weeks $(9 \cdot 1-20 \cdot 0)$ in the panitumumab group and $15 \cdot 0$ weeks $(7 \cdot 0-19 \cdot 9)$ in the control group.

Median overall survival was 11.1 months (95\% CI $9 \cdot 8-12 \cdot 2)$ in the panitumumab group and $9 \cdot 0$ months 


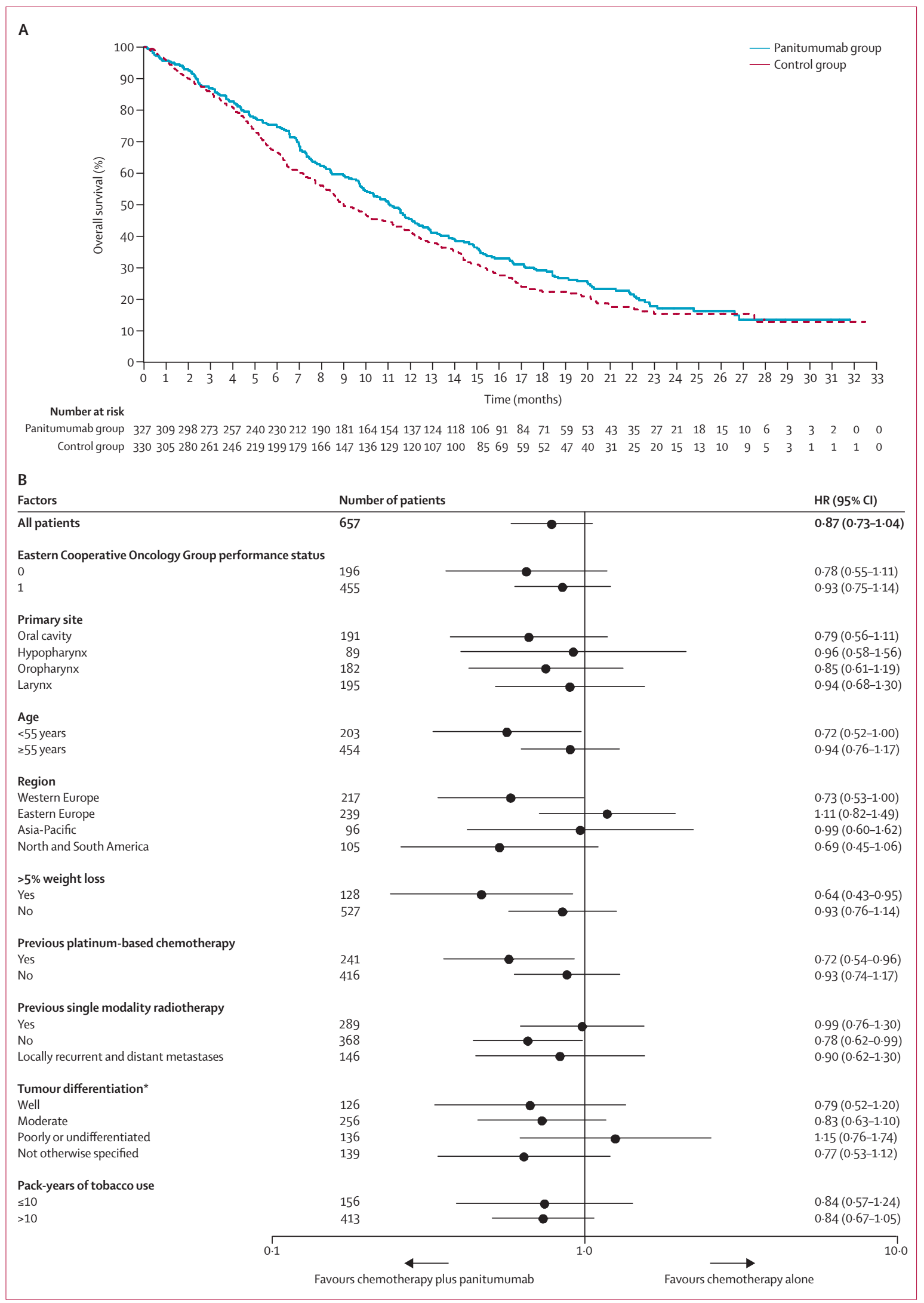




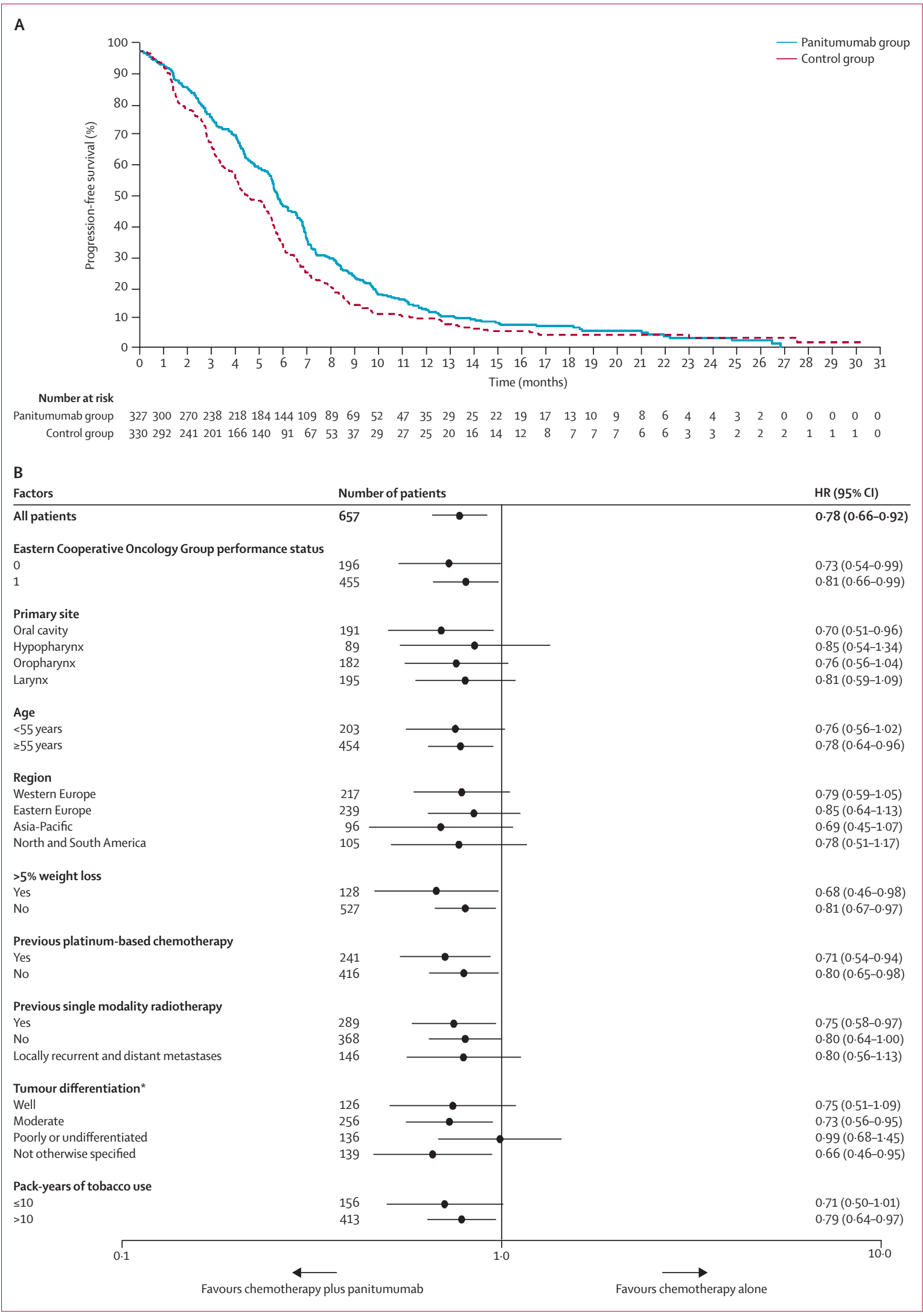

Figure 3: Kaplan-Meier curves by group (A) and subgroup analyses (B) for progressionfree survival

*From local pathologist assessments on case report form. 
$(8 \cdot 1-11 \cdot 2)$ in the control group $(\mathrm{p}=0 \cdot 1403$; HR $0 \cdot 873,95 \%$ CI 0.729-1.046; figure 2). Median follow-up was 44.0 weeks (IQR 21.0-75.0) in the panitumumab group and 35.0 weeks $(16 \cdot 0-66 \cdot 0)$ in the control group. At the time of analysis, 242 patients $(74 \%)$ in the panitumumab

\begin{tabular}{|lcc|}
\hline & Panitumumab group ( $\mathbf{n}=327)$ & Control group ( $\mathbf{n = 3 3 0 )}$ \\
\hline Included in tumour response analysis & $278(85 \%)$ & $288(87 \%)$ \\
Complete response & $5(2 \%)$ & $5(2 \%)$ \\
Partial response & $96(35 \%)$ & $68(24 \%)$ \\
Stable disease & $128(46 \%)$ & $134(47 \%)$ \\
Disease progression & $18(6 \%)$ & $34(12 \%)$ \\
Not assessable & $2(1 \%)$ & $3(1 \%)$ \\
Not done & $29(10 \%)$ & $44(15 \%)$ \\
Objective response (complete response and & $101(36 \%, 31-42)$ & $73(25 \%, 20-31)$ \\
partial response) & & $207(72 \%, 66-77)$ \\
Disease control (complete response, partial & $229(82 \%, 77-87)$ & $6 \cdot 7(5 \cdot 9-11 \cdot 3)$ \\
response, and stable disease) & $6 \cdot 1(5 \cdot 9-11 \cdot 0)$ & $5 \cdot 7(4 \cdot 7-6 \cdot 2)$ \\
Time to response (weeks) & $5 \cdot 6(4 \cdot 8-6 \cdot 2)$ & \\
Duration of response (months) & & \\
Data are $n$ (\%), $n$ (\%, 95\% Cl), or median (IQR). *Included only patients who had an objective response. \\
\hline Table 2: Best overall response
\end{tabular}

group and 241 (73\%) in the control group had died We assessed the proportional hazards assumption between the treatment groups with graphical and numerical methods based on cumulative Martingale residuals and recorded no evidence of non-proportionality $(\mathrm{p}=0 \cdot 16)$.

Median PFS was $5 \cdot 8$ months $(95 \%$ CI 5.6-6.6) in the panitumumab group and 4.6 months $(4 \cdot 1-5 \cdot 4)$ in the control group (HR 0.780, 95\% CI 0.659-0.922; $\mathrm{p}=0.0036$; figure 3). At the time of analysis, 290 patients $(89 \%)$ in the panitumumab group and 275 (83\%) in the control group had progressed or died. Sensitivity analyses suggested minor non-proportionality between treatment groups for PFS, but the results were consistent with those from the primary analysis (data not shown).

Overall, 566 patients had at least one baseline radiologically one-dimensionally measurable lesion. The proportions of participants who had an objective response (odds ratio $1 \cdot 69,95 \%$ CI $1 \cdot 15-2 \cdot 44 ; p=0 \cdot 0065$ ) or achieved disease control (odds ratio 1.84, 1.21-2.81; $\mathrm{p}=0 \cdot 0038$ ) were significantly higher in the panitumumab group than in the control group (table 2). Median DOR and median TTR were similar in the two groups (table 2).

Subgroup analyses of overall survival and PFS suggested that the results for overall survival (figure 2)

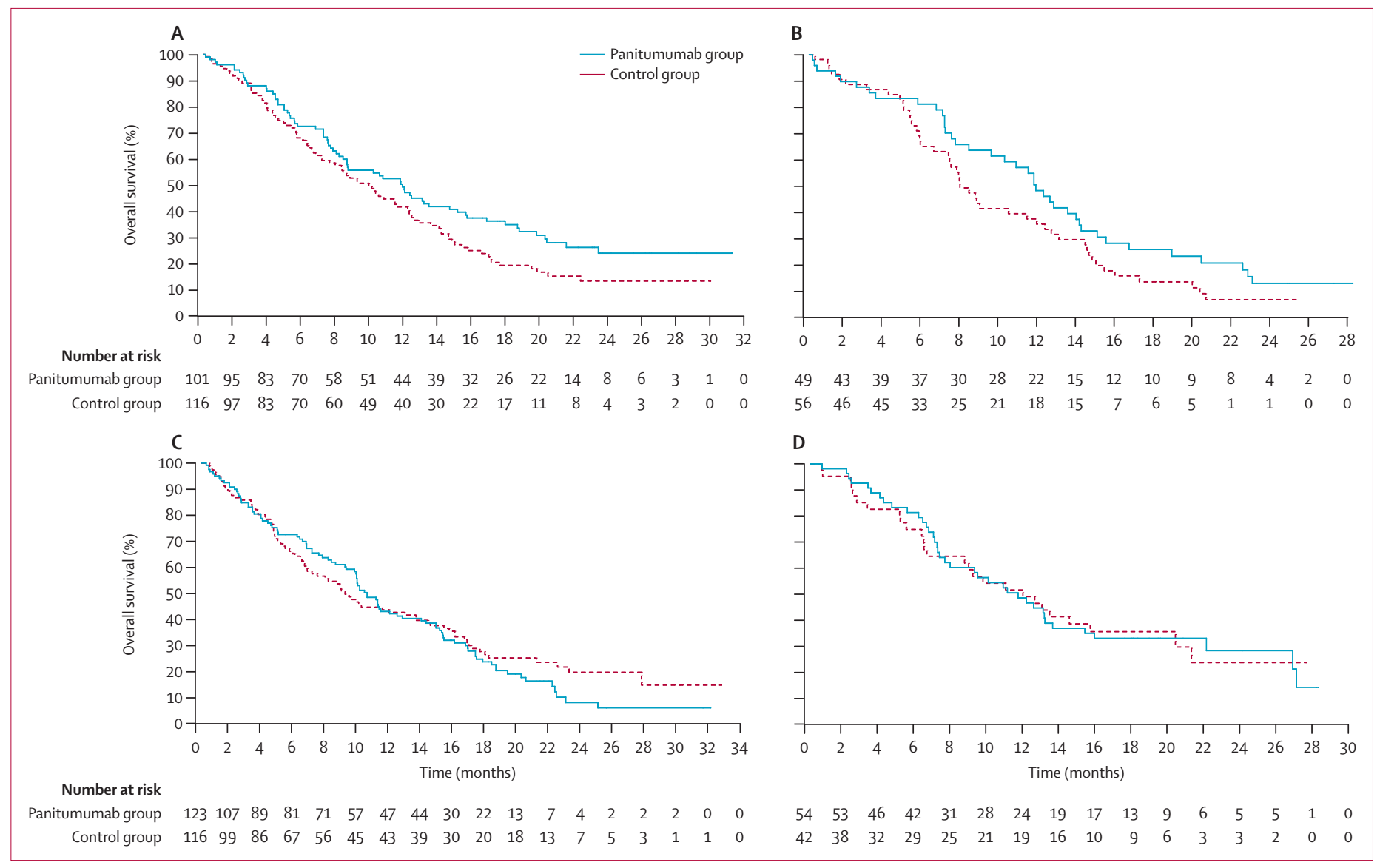

Figure 4: Overall survival by group in (A) western Europe, (B) North and South America, (C) eastern Europe, and (D) the Asia-Pacific region 


\begin{tabular}{|c|c|c|c|c|c|c|}
\hline & \multicolumn{2}{|c|}{ Panitumumab group } & \multicolumn{2}{|l|}{ Control group } & \multirow[t]{2}{*}{ Hazard ratio } & \multirow{2}{*}{$\begin{array}{l}\text { Log-rank } \\
\text { p value }\end{array}$} \\
\hline & Died & Overall survival (months) & Died & Overall survival (months) & & \\
\hline Western Europe & 69/101 (68\%) & $11 \cdot 7(8 \cdot 4-14 \cdot 9)$ & $86 / 116(74 \%)$ & $9 \cdot 7(7 \cdot 4-12 \cdot 1)$ & 0.727 (0.529-0.999) & 0.0478 \\
\hline $\begin{array}{l}\text { North and South } \\
\text { America }\end{array}$ & $39 / 49(80 \%)$ & $11 \cdot 7(8 \cdot 2-13 \cdot 9)$ & $47 / 56(84 \%)$ & $7 \cdot 8(6 \cdot 4-11 \cdot 7)$ & $0.692(0.450-1.065)$ & 0.0914 \\
\hline Eastern Europe & $96 / 123(78 \%)$ & $10 \cdot 3(9 \cdot 6-12 \cdot 6)$ & $81 / 116(70 \%)$ & $9 \cdot 0(6 \cdot 6-13 \cdot 4)$ & $1.108(0.823-1.492)$ & 0.5004 \\
\hline Asia-Pacific region & $38 / 54(70 \%)$ & $11 \cdot 5(7 \cdot 4-15 \cdot 1)$ & $27 / 42(64 \%)$ & $11 \cdot 7(6 \cdot 4-20 \cdot 1)$ & $0.985(0.600-1.616)$ & 0.9526 \\
\hline
\end{tabular}

and PFS (figure 3) were consistent across most patient subgroups. Of note, median overall survival and HRs varied by geographic region (figure 4 , table 3 ).

In univariate and multivariate analyses, several baseline covariates (such as previous platinum chemotherapy, ECOG performance status, weight loss in the previous 6 months, and disease stage) were significantly associated with overall survival (appendix). The effect of panitumumab in the multivariate model (HR $0 \cdot 875$, 95\% CI 0.731-1.048; $\mathrm{p}=0 \cdot 146$ ) was consistent with that in the primary analysis (appendix).

Grade 3 or 4 treatment-emergent adverse events were generally more common in the panitumumab group than in the control group (table 4). Differences between groups were significant for skin or eye toxicity $(\mathrm{p}<0 \cdot 0001)$; hypomagnesaemia $(\mathrm{p}<0.0001)$; cardiac arrhythmias $(\mathrm{p}=0 \cdot 0474)$; and diarrhoea $(\mathrm{p}=0 \cdot 0175)$. Grade 3 or 4 infusion reactions were rare (table 4). Serious adverse events occurred in 157 (48\%) of 325 patients in the panitumumab group included in the safety analyses and $139(43 \%)$ of 325 in the control group. Adverse events resulting in discontinuation of panitumumab or chemotherapy, or both, or removal from the study occurred in 91 patients (28\%) in the panitumumab group and 78 patients $(24 \%)$ in the control group. Of these patients, 69 patients in the panitumumab group and 59 in the control group discontinued because of treatment-related toxicity.

Overall, 48 (15\%) of the 325 patients in the panitumumab group and 41 (13\%) of 325 in the control group included in safety analyses had on-treatment fatal adverse events. Death and disease progression occurred simultaneously in 12 patients in the panitumumab group and 17 patients in the control group. Other fatal adverse events were cardiac events (eight patients [2\%] in the panitumumab group; seven [2\%] in the control group), sepsis or febrile neutropenia (four [1\%]; three [1\%]), and haemorrhage (two [1\%]; five [2\%]). By investigator attribution, treatmentrelated deaths occurred in 14 patients $(4 \%)$ in the panitumumab group and eight (2\%) in the control group. Five patients $(2 \%)$ had fatal adverse events that were attributed to panitumumab by the investigators: myocardial infarction, cardiac failure, cerebrovascular accident, acute renal failure, and haemorrhagic diarrhoea.

\begin{tabular}{|c|c|c|c|c|}
\hline & \multicolumn{2}{|c|}{ Panitumumab group $(n=325)$} & \multicolumn{2}{|c|}{ Control group $(n=325)$} \\
\hline & Grade 3 or 4 & Grade 4 & Grade 3 or 4 & Grade 4 \\
\hline Patients with any event & $219(67 \%)$ & $96(30 \%)$ & $214(66 \%)$ & $81(25)$ \\
\hline \multicolumn{5}{|l|}{ Adverse events of interest } \\
\hline Skin or eyes, or both* & $62(19 \%)$ & $5(2 \%)$ & $6(2 \%)$ & 0 \\
\hline Hypomagnesaemia & $40(12 \%)$ & $15(5 \%)$ & $12(4 \%)$ & $4(1 \%)$ \\
\hline Hypokalaemia & $33(10 \%)$ & $9(3 \%)$ & $23(7 \%)$ & $10(3 \%)$ \\
\hline Stomatitis or oral mucositis & $29(9 \%)$ & $2(1 \%)$ & $28(9 \%)$ & $2(1 \%)$ \\
\hline Cardiac arrhythmias & $19(6 \%)$ & $3(1 \%)$ & $8(2 \%)$ & $5(2 \%)$ \\
\hline Dehydration & $16(5 \%)$ & $2(1 \%)$ & $7(2 \%)$ & 0 \\
\hline Diarrhoea & $15(5 \%)$ & $1(<1 \%)$ & $4(1 \%)$ & $1(<1 \%)$ \\
\hline Venous embolic and thrombotic events & $13(4 \%)$ & $8(2 \%)$ & $6(2 \%)$ & $4(1 \%)$ \\
\hline Acute renal failure & $10(3 \%)$ & $3(1 \%)$ & $11(3 \%)$ & $1(<1 \%)$ \\
\hline Hypocalcaemia & $8(2 \%)$ & $5(2 \%)$ & $7(2 \%)$ & $2(1 \%)$ \\
\hline $\begin{array}{l}\text { Embolic and thrombotic events } \\
\text { (unspecified or mixed vessel type) }\end{array}$ & $7(2 \%)$ & $3(1 \%)$ & $5(2 \%)$ & $1(<1 \%)$ \\
\hline Arterial embolic and thrombotic events & $5(2 \%)$ & $3(1 \%)$ & $1(<1 \%)$ & $1(<1 \%)$ \\
\hline Infusion reaction $†$ & $2(1 \%)$ & 0 & 0 & 0 \\
\hline Ischaemic heart disease & $2(1 \%)$ & 0 & 0 & 0 \\
\hline Interstitial lung disease & $1(<1 \%)$ & $1(<1 \%)$ & $1(<1 \%)$ & 0 \\
\hline Severe cutaneous adverse reactions & $1(<1 \%)$ & $1(<1 \%)$ & 0 & 0 \\
\hline Impaired or delayed wound healing & 0 & 0 & $1(<1 \%)$ & $1(<1 \%)$ \\
\hline Haematological toxicity & $141(43 \%)$ & $64(20 \%)$ & $147(45 \%)$ & $55(17 \%)$ \\
\hline Neutropenia & $103(32 \%)$ & $43(13 \%)$ & $106(33 \%)$ & $40(12 \%)$ \\
\hline Anaemia & $39(12 \%)$ & $5(2 \%)$ & $47(14 \%)$ & $5(2 \%)$ \\
\hline Thrombocytopenia & $21(6 \%)$ & $9(3 \%)$ & $25(8 \%)$ & $8(2 \%)$ \\
\hline Febrile neutropenia & $20(6 \%)$ & $12(4 \%)$ & $17(5 \%)$ & $6(2 \%)$ \\
\hline \multicolumn{5}{|c|}{$\begin{array}{l}\text { Adverse events were coded using Medical Dictionary for Regulatory Activities (version 13.0) and graded according to } \\
\text { National Cancer Institute Common Terminology Criteria for Adverse Events (version 3.0), with the exception of some } \\
\text { dermatological or skin adverse events that were graded with modifications to the criteria. }{ }^{*} \text { Includes preferred terms } \\
\text { considered related to skin and eye toxicity. †Per US prescribing information. }\end{array}$} \\
\hline
\end{tabular}

Fatal adverse events within the first 30 days of treatment occurred in 14 patients (4\%) in the panitumumab group and 13 patients (4\%) in the control group.

Of 298 patients who received panitumumab and for whom serum samples were available for testing, three $(1 \%)$ developed anti-panitumumab antibodies. However, no anti-panitumumab-neutralising antibodies were detected.

Fixed-formalin paraffin-embedded tumour samples were available for assessment of p16 status for 443 (67\%) 
of 657 patients, yielding 1083 evaluable tumour cores. $99(22 \%)$ of these 443 had p16-positive tumours and $344(78 \%)$ had p16-negative tumours (table 5, appendix). The cumulative frequency distribution of p16-positive samples is shown in the appendix. The proportion of patients with p16-positive tumours was similar in the two

\begin{tabular}{|c|c|c|c|c|c|c|}
\hline & \multirow{2}{*}{$\begin{array}{l}\text { Assessable } \\
\text { for p16 } \\
(n=443)\end{array}$} & \multirow{2}{*}{$\begin{array}{l}\text { Not } \\
\text { assessable } \\
\text { for p16 } \\
(n=214)\end{array}$} & \multicolumn{2}{|c|}{ p16 positive $(n=99)$} & \multicolumn{2}{|c|}{ p16 negative $(n=344)$} \\
\hline & & & $\begin{array}{l}\text { Panitumumab } \\
\text { group }(\mathrm{n}=57)\end{array}$ & $\begin{array}{l}\text { Control } \\
\text { group } \\
(\mathrm{n}=42)\end{array}$ & $\begin{array}{l}\text { Panitumumab } \\
\text { group }(n=179)\end{array}$ & $\begin{array}{l}\text { Control } \\
\text { group } \\
(n=165)\end{array}$ \\
\hline Hypopharynx & $61(14 \%)$ & $28(13 \%)$ & $5(9 \%)$ & $3(7 \%)$ & $34(19 \%)$ & $19(12 \%)$ \\
\hline Oral cavity & $119(27 \%)$ & $72(34 \%)$ & $12(21 \%)$ & $6(14 \%)$ & $49(27 \%)$ & $52(32 \%)$ \\
\hline Larynx & $137(31 \%)$ & $58(27 \%)$ & $16(28 \%)$ & $10(24 \%)$ & $58(32 \%)$ & $53(32 \%)$ \\
\hline Oropharynx & $126(28 \%)$ & $56(26 \%)$ & $24(42 \%)$ & $23(55 \%)$ & $38(21 \%)$ & $41(25 \%)$ \\
\hline
\end{tabular}

groups: 57 (24\%) of 236 in the panitumumab group, and $42(20 \%)$ of 207 in the control group (table 4). Many p16 positive patients had oropharyngeal tumours (table 4). The proportions of patients with different sites of tumour origin were generally consistent between patients who could and could not be assessed for p16 status (table 4). Demographic and baseline characteristics were generally balanced between treatment groups in p16-negative and p16-positive patients (data not shown). Furthermore, the proportions of patients who had previously received platinum-based chemotherapy differed between groups for both p16-negative (73 [41\%] of 179 in the panitumumab group vs 55 [33\%] of 165 in the control group) and p16positive patients (21 [37\%] of 57 patients vs 21 [50\%] of 42$)$.

Median overall survival in patients with p16-negative tumours was longer in the panitumumab group than in the control group, but not in those with p16-positive tumours (figure 5, table 6). Similarly, median PFS in patients with p-16 negative tumours was longer in the panitumumab group than in the control group, but not in those with p16-positive tumours (figure 5, table 6).

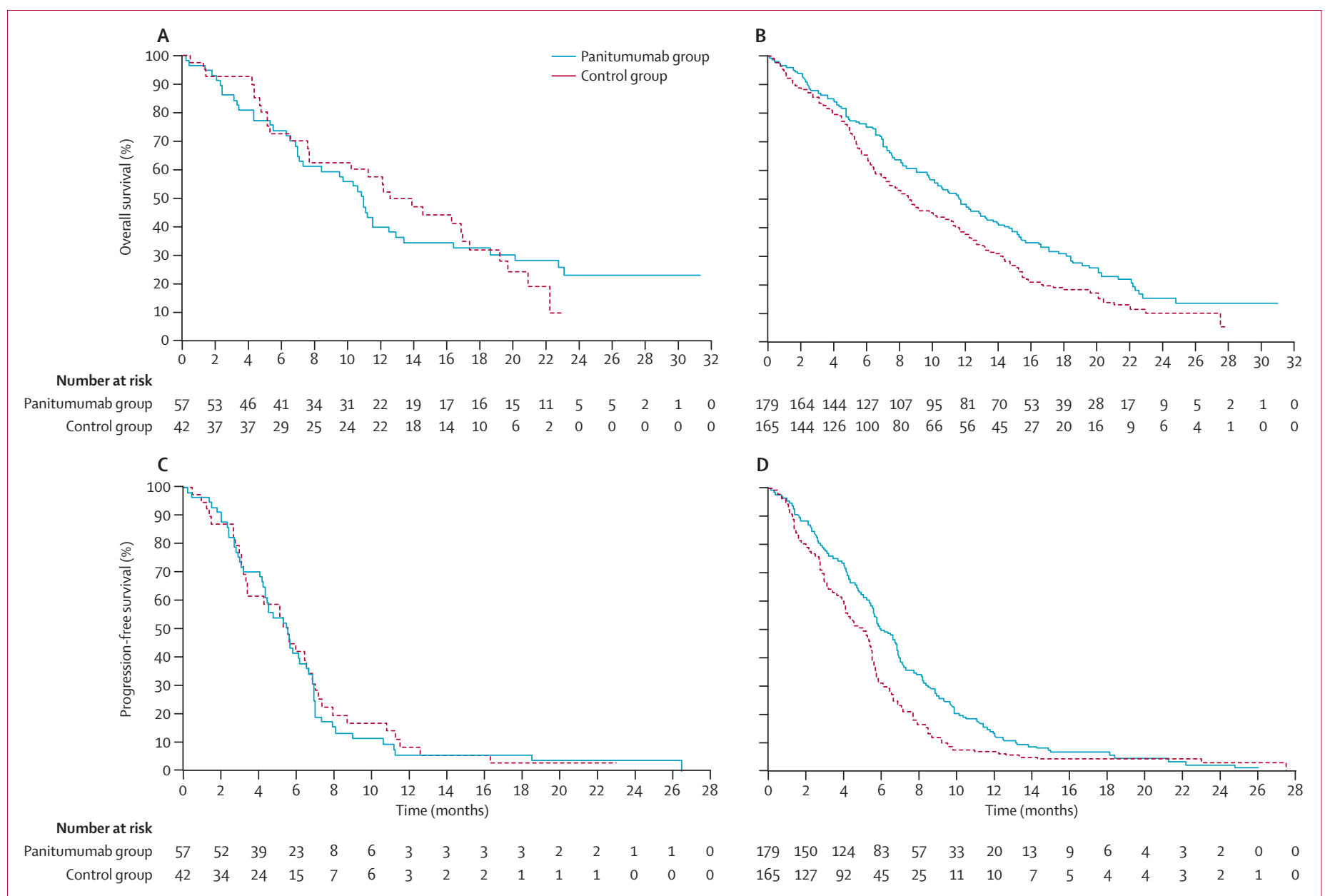

Figure 5: Overall and progression-free survival by $\mathrm{p} 16$ status

Overall survival in p16-positive (A) and p16-negative (B) patients. Progression-free survival in p16-positive (C) and p16-negative (D) patients. p16=p16-INK4A. 


\begin{tabular}{|c|c|c|c|c|c|c|c|c|}
\hline & \multicolumn{4}{|c|}{ p16-positive patients } & \multicolumn{4}{|c|}{ p16-negative patients } \\
\hline & $\begin{array}{l}\text { Panitumumab } \\
\text { group }\end{array}$ & Control group & Hazard ratio & $\begin{array}{l}\text { Log-rank } \\
\text { p value }\end{array}$ & $\begin{array}{l}\text { Panitumumab } \\
\text { group }\end{array}$ & Control group & Hazard ratio & $\begin{array}{l}\text { Log-rank } \\
\text { p value }\end{array}$ \\
\hline Died & $42 / 57(74 \%)$ & $30 / 42(71 \%)$ &.. &.. & $133 / 179(74 \%)$ & $133 / 165(81 \%)$ &.. &.. \\
\hline $\begin{array}{l}\text { Overall survival } \\
\text { (months) }\end{array}$ & $11 \cdot 0(7 \cdot 3-12 \cdot 9)$ & $12 \cdot 6(7 \cdot 7-17 \cdot 4)$ & $1.00(0.62-1.61)$ & 0.998 & $11 \cdot 7(9 \cdot 7-13 \cdot 7)$ & $8.6(6 \cdot 9-11 \cdot 1)$ & $0.73(0.58-0.93)$ & 0.0115 \\
\hline Progressed & $54 / 57(95 \%)$ & $36 / 42(86 \%)$ & .. & .. & $160 / 179(89 \%)$ & $148 / 165(90 \%)$ & .. & .. \\
\hline $\begin{array}{l}\text { Progression-free } \\
\text { survival (months) }\end{array}$ & $5 \cdot 6(4 \cdot 4-6 \cdot 5)$ & $5 \cdot 5(3 \cdot 4-6 \cdot 7)$ & $1.08(0.71-1.65)$ & 0.730 & $6 \cdot 0(5 \cdot 6-6 \cdot 9)$ & $5 \cdot 1(4 \cdot 1-5 \cdot 5)$ & $0.69(0.55-0.87)$ & 0.0013 \\
\hline
\end{tabular}

Of patients in the control group, those who were p16 positive had numerically, but not statistically significantly, longer median overall survival than did those who were p16 negative $(12.6$ months $[7.7-17.4]$ vs 8.6 months [6.9-11·1]; HR 0.70, 95\% CI 0.47-1.04; figure 6). Toxicity was generally similar between p16-negative and p16positive patients, and between patients of different p16 status in the two treatment groups (appendix).

\section{Discussion}

SPECTRUM was a global study of a geographically diverse population of patients with wide variation in previous treatment. The results show that the addition of panitumumab to a regimen of cisplatin and fluorouracil does not significantly improve overall survival of patients with recurrent or metastatic SCCHN. By contrast, both progression-free survival and frequency of objective response were significantly improved by the addition of panitumumab, confirming its antitumour activity in SCCHN. Furthermore, in a prospectively defined retrospective analysis of p16 tumour status, we showed that overall survival was longer for p16-positive patients than for p16-negative patients who received only cisplatin and fluorouracil, suggesting that p16 status could be used as a prognostic marker in recurrent or metastatic SCCHN. Notably, the addition of panitumumab to the regimen resulted in significant improvements in overall and progression-free survival in patients with p16negative tumours, but not in those with p16-positive tumours.

Two features of the primary analysis are noteworthy. First, median overall survival in both groups was longer than in two randomised studies. ${ }^{6,9}$ Median overall survival and progression-free survival in the group given cisplatin and fluorouracil alone were unexpectedly better than those in the previous studies (overall survival range 7.4-8.0 months; progression-free survival $2 \cdot 7-3 \cdot 3$ months). ${ }^{6,9}$ Second, we recorded regional variation in the effect of panitumumab on overall survival, which potentially affected the aggregate results. The inclusion of patients from the Asia-Pacific region (who had fairly long overall survival) might account, at least partly, for the extended overall survival in the control

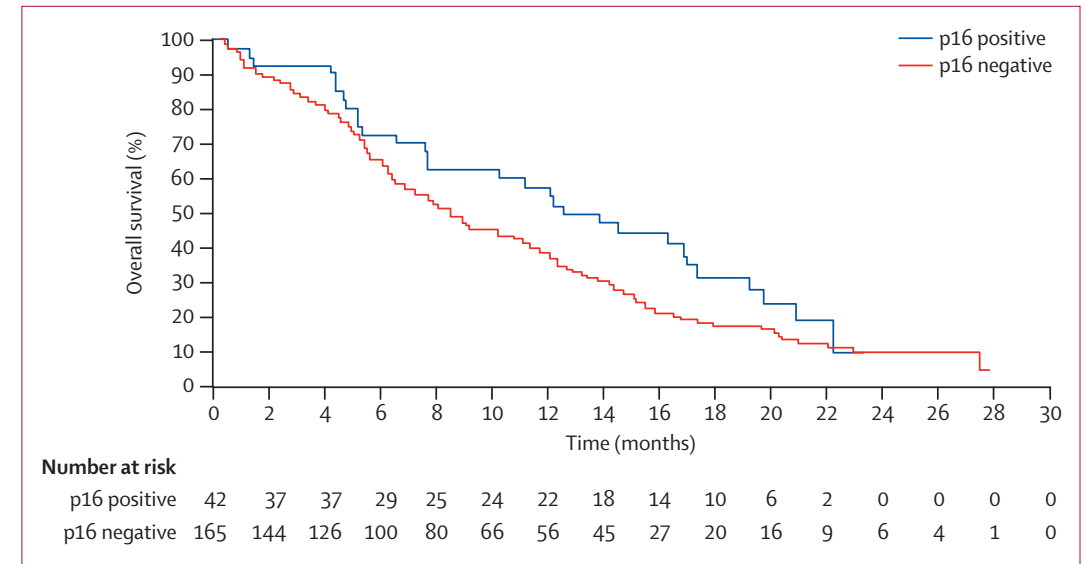

Figure 6: Overall survival in control group by $\mathrm{p} 16$ status p16=p16-INK4A.

group compared with that in EXTREME $^{9}(9 \cdot 0$ months vs 7.4 months).

We showed numerically, but not significantly, longer overall survival in the group given panitumumab for most subgroups. We recorded suggestions of increased benefits in subgroups known to have poor outlooks, such as those who had greater than $5 \%$ weight loss, previous platinum exposure, and moderately and well differentiated tumour subtypes. Progression-free survival was longer for patients who received panitumumab in most subgroups. The univariate and multivariate prognostic factors for overall survival identified in our study (performance status at study entry, weight loss, and previous platinum chemotherapy) were consistent with those previously reported by Argiris and colleagues..$^{35}$

The HPV analysis, in which p16 immunohistochemistry was used as a surrogate marker, showed that a p16positive status was a prognostic factor. This finding is especially noteworthy because it extends results of previous studies in which tumour HPV status was shown to have prognostic value in patients with locoregionally advanced oropharyngeal SCCHN treated with chemotherapy or radiotherapy, or both. ${ }^{11-16}$ In our study, about half of p16-positive tumours were oropharyngeal, and the rest of p16-positive patients had laryngeal, 
hypopharyngeal, and oral tumours, which is consistent with previous findings. ${ }^{36,37}$ The relative importance of HPV status in different SCCHN sites of origin remains to be defined in future clinical investigation. The proportion of p16-positive patients defined with our prespecified definition of strong and diffuse nuclear and cytoplasmic staining in at least $10 \%$ of tumour cells was also consistent with previous studies; ${ }^{36,37}$ results were similar when alternative cutoffs were used.

Notably, we showed that tumour p16 status might be a predictive biomarker for overall and progression-free survival in patients with recurrent or metastatic SCCHN treated with an anti-EGFR monoclonal antibody combined with chemotherapy. Furthermore, our results suggest that, in addition to the apparent effects of regional variation, variation in tumour p16 status could have affected the aggregate outcome results. The randomisation stratification of patients by combined tumour site (hypopharynx or oral cavity vs oropharynx or larynx) might have resulted in unequal stratification of patients with p16-positive tumours (most of whom had oropharyngeal tumours) between the two groups.

Our results raise important questions about tumour HPV status as a potential predictive biomarker in recurrent or metastatic SCCHN. SCCHN associated with HPV infection seems to be a biologically distinct subset of SCCHN, particularly with regard to differences in genetic alterations between HPV-positive and HPV-negative

\section{Panel: Research in context}

\section{Systematic review}

We did not do a formal systematic analysis before the start of our trial. We carefully reviewed reports of clinical trials assessing platinum-based chemotherapy treatment in recurrent or metastatic squamous-cell carcinoma of the head and neck (SCCHN) that were identified by a search of PubMed and abstracts from international clinical oncology meetings. The review showed that overall survival in this population remains poor despite presently available treatments and that anti-EGFR agents might have activity in this setting. On the basis of this review and discussions with researchers and clinical oncologists, improvement in overall response and overall survival was evidently a realistic goal for a clinical trial in this population of patients.

\section{Interpretation}

As far as we are aware, SPECTRUM is the largest global study of an EGFR inhibitor in combination with platinum and high-intensity infusional fluorouracil chemotherapy in a diverse population of patients with recurrent or metastatic SCCHN. Overall survival did not significantly improve with the addition of panitumumab to the chemotherapy regimen, but we did record improvements in progression-free survival and objective response. Furthermore, in a retrospective analysis, a negative tumour human papillomavirus status, assessed by 16 immunohistochemistry, predicted overall and progression-free survival after treatment with cisplatin and fluorouracil plus panitumumab. Moreover, a p16-positive status was a favourable prognostic marker in patients who received only chemotherapy, suggesting a potential prognostic effect in this population of patients. Our results indicate that tumour p16 status, regional differences in overall survival, and other factors (eg, the intensity and amount of previous treatment) might be important considerations in the design of future global trials in recurrent or metastatic SCCHN. disease. ${ }^{11,13,17,18}$ When tested as a specific immunohistochemical marker for HPV oncogene expression, p16 with a $10 \%$ cutoff is robust, ${ }^{38}$ and has high concordance with high-risk HPV DNA, RNA, and E6 and E7 gene expression. ${ }^{39-41}$ Immunohistochemical detection of p16 has been successfully used in several clinical studies of patients with SCCHN as a screening marker to estimate HPV status. ${ }^{12,41-43}$ However, genetic differences between SCCHN types might affect the predictive ability of p16 as a biomarker. ${ }^{44}$ We assessed p16 status as a biomarker by tumour site of origin. In view of the number of patients with each of the tumour types in our study, further research will be required to resolve the issue of genetic variation.

The results for overall survival in our primary analysis are surprising when compared with the phase 3 EXTREME study, ${ }^{9}$ in which overall survival was improved in patients who received cetuximab plus cisplatin or carboplatin and fluorouracil compared with those who did not receive cetuximab $(10.1$ vs 7.4 months; HR 0.80 ; $\mathrm{p}=0 \cdot 04)$. Although heterogeneity in populations of patients makes comparisons between trials difficult, differences between the two studies in design and patient eligibility criteria could explain, at least partly, the varying findings. First, in the EXTREME study, ${ }^{9}$ continuation of cetuximab monotherapy after six cycles of chemotherapy was mandatory for patients who had not experienced disease progression, whereas in our trial, continuation of panitumumab monotherapy was optional.

Second, treatment received before enrolment differed greatly between the two studies (previous chemotherapy: $81 \%$ in our study vs $38 \%$ in EXTREME ${ }^{9}$ ). In our study, patients were not stratified by type of previous treatment, which also varied by region. Moreover, patients in EXTREME were allowed to receive either cisplatin or carboplatin from enrolment, whereas in our study, patients had to begin cisplatin and could only switch to carboplatin for reduced creatinine clearance $(<50 \mathrm{~mL} / \mathrm{min})$ or grade 2 neurotoxicity. This requirement could have resulted in enrolment of a population with improved performance status, and therefore longer overall survival, compared with EXTREME.'

Third, in the EXTREME study, ${ }^{9}$ most patients were recruited from western Europe, whereas we recruited worldwide, with a third of patients from western Europe. In a subgroup analysis, we showed that overall survival was longer in patients from western Europe who received panitumumab than in those who did not.

Fourth, overall survival, but not progression-free survival, in our trial might have been confounded by treatment given after progression, including cytotoxic chemotherapy and targeted agents. Although roughly $5 \%$ of patients who received panitumumab plus chemotherapy and $9 \%$ of those who received chemotherapy alone received subsequent anti-EGFR targeted therapy for disease progression, the fairly infrequent crossover suggests that this potential confounder had a small effect. Finally, unlike in 
EXTREME, ${ }^{9}$ we did not insist that all patients had to have disease that could be measured with RECIST at enrolment, which was appropriate because the primary endpoint was overall survival and means the results can be generalised to a broad population.

Notably, overall survival of p16-negative patients in our study was longer than that in EXTREME $(10 \cdot 1$ months in group given chemotherapy plus cetuximab; 7.4 months in group given chemotherapy alone). ${ }^{9}$ The results of a retrospective analysis of tumour p16 status in EXTREME, ${ }^{45}$ which are qualitatively different from ours, suggested that the survival benefit of cetuximab treatment was independent of tumour p16 status. However, only $44(12 \%)$ of 381 assessable patients in EXTREME were identified as being p16 positive, which could have restricted the ability to draw meaningful conclusions about the outcomes in these patients. Nevertheless, these contradictory findings underscore the necessity to further assess the role of HPV in the setting of recurrent or metastatic disease (panel). Prospective assessment will be necessary to confirm tumour HPV status as a predictive biomarker for anti-EGFR therapy in recurrent or metastatic SCCHN.

We noted no unexpected safety findings. Frequency of skin toxicity, hypomagnesaemia, hypokalaemia, diarrhoea, and dehydration were generally consistent with that reported with other anti-EGFR monoclonal antibodies in patients with recurrent or metastatic SCCHN. ${ }^{9,46}$ These events have also been reported in patients receiving panitumumab with or without chemotherapy for metastatic colorectal cancer. $^{47-49}$ The subjectivity of grading of skin toxicity, the use of different grading scales, and differences in the descriptive terms for skin toxicity between our trial and EXTREME 9 make comparisons of the frequency of skin toxicity between the two studies difficult.

Compliance with chemotherapy in both groups in our study was good. Exposure to cisplatin or carboplatin was similar across the groups, and exposure to fluorouracil was moderately greater in the group that received chemotherapy plus panitumumab (probably because of the reduced disease progression rate).

In conclusion, the addition of panitumumab to chemotherapy did not improve overall survival of patients with recurrent or metastatic SCCHN. However, significant improvements were noted with the addition of panitumumab in terms of PFS and the number of patients who achieved an objective response. Subgroup analyses suggested that clinical benefit may have been greater among certain patient subgroups, although these data should be interpreted with care given the small size of some of the groups. Furthermore, our retrospective analyses suggested that tumour p16 status might have both prognostic and predictive value in patients with recurrent or metastatic SCCHN treated with panitumumab combined with chemotherapy, although these findings require further validation.

\section{Contributors}

JBV did the literature search for the study. JS-W, ID, LL, CV, PF, SR, MT, VRP, SF, CRB, and BAB collected data. JS-W, EW, PF, SR, MT, VRP, SF, $\mathrm{CRB}, \mathrm{AAF}, \mathrm{BNS}$, and $\mathrm{BAB}$ enrolled patients and obtained study materials. JBV, JS-W, and ZP designed the study. JBV, JS-W, LL, EW, MT, VRP, SF, CRB, BNS, KSO, ZP, and BAB analysed and interpreted data. JBV, JS-W, EW, CV, PF, SR, MT, SF, CRB, AAF, BNS, KSO, ZP, and BAB wrote the report. KS participated in the steering committee. KSO developed and implemented the biomarker testing strategy. BAB provided administrative support and helped to obtain financial support for the study.

\section{Conflicts of interest}

JBV has served as a paid consultant for Amgen Inc and has received honoraria from Amgen Inc for scientific presentations at satellite symposia and for advisory boards. JS-W has received honoraria for advisory boards and speaking, travel support, and research funding from Amgen Inc, Roche, Eli Lilly, Boehringer Ingelheim, and Merck Serono. LL has received honoraria for advisory boards from Amgen Inc. MT has received research funding from Eisai and Yakult. SF has served as a paid consultant for, and has received honoraria for participating in a steering committee, from Amgen Inc. KSO, ZP, and BAB are employees of, and own stock in, Amgen Inc. The other authors declare that they have no conflicts of interest.

\section{Acknowledgments}

We thank Benjamin Scott and Ali Hassan (Complete Healthcare Communications, Chadds Ford, PA, USA; funded by Amgen Inc) for assistance in the writing of this report.

\section{References}

1 National Comprehensive Cancer Network. NCCN clinical practice guidelines in oncology: head and neck cancers, version 1.2009. Fort Washington, PA: National Comprehensive Cancer Network, 2008.

2 Jacobs C, Lyman G, Velez-Garcia E, et al. A phase III randomized study comparing cisplatin and fluorouracil as single agents and in combination for advanced squamous cell carcinoma of the head and neck. J Clin Oncol 1992; 10: 257-63.

3 Forastiere AA, Metch B, Schuller DE, et al. Randomized comparison of cisplatin plus fluorouracil and carboplatin plus fluorouracil versus methotrexate in advanced squamous-cell carcinoma of the head and neck: a Southwest Oncology Group study. J Clin Oncol 1992; 10: 1245-51.

4 Clavel M, Vermorken JB, Cognetti F, et al. Randomized comparison of cisplatin, methotrexate, bleomycin and vincristine (CABO) versus cisplatin and 5-fluorouracil (CF) versus cisplatin (C) in recurrent or metastatic squamous cell carcinoma of the head and neck. A phase III study of the EORTC Head and Neck Cancer Cooperative Group. Ann Oncol 1994; 5: 521-26.

5 Gibson MK, Li Y, Murphy B, et al. Randomized phase III evaluation of cisplatin plus fluorouracil versus cisplatin plus paclitaxel in advanced head and neck cancer (E1395): an intergroup trial of the Eastern Cooperative Oncology Group. J Clin Oncol 2005; 23: 3562-67.

6 Burtness B, Goldwasser MA, Flood W, Mattar B, Forastiere AA. Phase III randomized trial of cisplatin plus placebo compared with cisplatin plus cetuximab in metastatic/recurrent head and neck cancer: an Eastern Cooperative Oncology Group study. J Clin Oncol 2005; 23: 8646-54.

7 Leemans CR, Braakhuis BJ, Brakenhoff RH. The molecular biology of head and neck cancer. Nat Rev Cancer 2011; 11: 9-22.

8 Sheu JJ, Hua CH, Wan L, et al. Functional genomic analysis identified epidermal growth factor receptor activation as the most common genetic event in oral squamous cell carcinoma. Cancer Res 2009; 69: 2568-76.

9 Vermorken JB, Mesia R, Rivera F, et al. Platinum-based chemotherapy plus cetuximab in head and neck cancer. N Engl J Med 2008; 359: 1116-27.

10 Langer CJ. Exploring biomarkers in head and neck cancer. Cancer 2012; 118: 3882-92.

11 Gillison ML, Koch WM, Capone RB, et al. Evidence for a causal association between human papillomavirus and a subset of head and neck cancers. J Natl Cancer Inst 2000; 92: 709-20. 
12 Ang KK, Harris J, Wheeler R, et al. Human papillomavirus and survival of patients with oropharyngeal cancer. N Engl J Med 2010; 363: 24-35.

13 Fakhry C, Westra WH, Li S, et al. Improved survival of patients with human papillomavirus-positive head and neck squamous cell carcinoma in a prospective clinical trial. J Natl Cancer Inst 2008; 100: 261-69.

14 Posner MR, Lorch JH, Goloubeva O, et al. Survival and human papillomavirus in oropharynx cancer in TAX 324: a subset analysis from an international phase III trial. Ann Oncol 2011; 22: 1071-77.

15 Rischin D, Young RJ, Fisher R, et al. Prognostic significance of p16INK4A and human papillomavirus in patients with oropharyngeal cancer treated on TROG 02.02 phase III trial. J Clin Oncol 2010; 28: 4142-48.

16 Ryerson AB, Peters ES, Coughlin SS, et al. Burden of potentially human papillomavirus-associated cancers of the oropharynx and oral cavity in the US, 1998-2003. Cancer 2008; 113: 2901-09.

17 Braakhuis BJ, Snijders PJ, Keune WJ, et al. Genetic patterns in head and neck cancers that contain or lack transcriptionally active human papillomavirus. J Natl Cancer Inst 2004; 96: 998-1006.

18 Leemans CR, Braakhuis BJ, Brakenhoff RH. The molecular biology of head and neck cancer. Nat Rev Cancer 2011; 11: 9-22.

19 Rampias T, Sasaki C, Weinberger P, Psyrri A. E6 and E7 gene silencing and transformed phenotype of human papillomavirus 16-positive oropharyngeal cancer cells. J Natl Cancer Inst 2009; 101: 412-23.

20 Kim SH, Koo BS, Kang S, et al. HPV integration begins in the tonsillar crypt and leads to the alteration of p16, EGFR and c-myc during tumor formation. Int J Cancer 2007; 120: 1418-25.

21 Kumar B, Cordell KG, Lee JS, et al. EGFR, p16, HPV Titer, Bcl-xL and p53, sex, and smoking as indicators of response to therapy and survival in oropharyngeal cancer. J Clin Oncol 2008; 26: 3128-37.

22 Young RJ, Rischin D, Fisher R, et al. Relationship between epidermal growth factor receptor status, p16(INK4A), and outcome in head and neck squamous cell carcinoma. Cancer Epidemiol Biomarkers Prev 2011; 20: 1230-37.

23 Hong A, Dobbins T, Lee CS, et al. Relationships between epidermal growth factor receptor expression and human papillomavirus status as markers of prognosis in oropharyngeal cancer. Eur J Cancer 2010; 46: 2088-96.

24 Perrone F, Suardi S, Pastore E, et al. Molecular and cytogenetic subgroups of oropharyngeal squamous cell carcinoma. Clin Cancer Res 2006; 12: 6643-51.

25 Peeters M, Cohn A, Kohne CH, Douillard JY. Panitumumab in combination with cytotoxic chemotherapy for the treatment of metastatic colorectal carcinoma. Clin Colorectal Cancer 2012; 11: $14-23$.

26 Kruser TJ, Armstrong EA, Ghia AJ, et al. Augmentation of radiation response by panitumumab in models of upper aerodigestive tract cancer. Int J Radiat Oncol Biol Phys 2008; 72: 534-42.

27 Wirth LJ, Posner MR, Haddad RI, et al. Phase I study of panitumumab + chemoradiotherapy (CRT) for head and neck cancer (HNC). Proc Am Soc Clin Oncol 2008; 26: 32360 (abstr).

28 Rowinsky EK, Schwartz GH, Gollob JA, et al. Safety, pharmacokinetics, and activity of ABX-EGF, a fully human anti-epidermal growth factor receptor monoclonal antibody in patients with metastatic renal cell cancer. J Clin Oncol 2004. 22: 3003-15.

29 Therasse P, Arbuck SG, Eisenhauer EA, et al. New guidelines to evaluate the response to treatment in solid tumors. J Natl Cancer Inst 2000; 92: 205-16.

30 Cancer Therapy Evaluation Program. Common Terminology Criteria for Adverse Events, version 3.0. Aug 9, 2006. http://ctep. cancer.gov/protocoldevelopment/electronic_applications/docs / ctcaev3.pdf (accessed May 9, 2013).

31 Lofgren JA, Dhandapani S, Pennucci JJ, et al. Comparing ELISA and surface plasmon resonance for assessing clinical immunogenicity of panitumumab. J Immunol 2007; 178: 7467-72.

32 Oliner KS, Ekdahl M, Kyshtoobayeva A, Bloom K, Bach BA. Qualification of a p16INK4A immunohistochemistry (IHC) assay for determination of HPV status in archival squamous cell carcinoma of the head and neck (SCCHN) formalin fixed paraffin embedded (FFPE) specimens from a randomized phase 3 trial. Cancer Res 2012; 72 (suppl 1): 554 (abstr).
33 Agresti A. Categorical Data Analysis. New York, NY: John Wiley and Sons, 1990.

34 Newcombe RG. Interval estimation for the difference between independent proportions: comparison of eleven methods. Stat Med 1998; 17: 873-890.

35 Argiris A, Li Y, Forastiere A. Prognostic factors and long-term survivorship in patients with recurrent or metastatic carcinoma of the head and neck. Cancer 2004; 101: 2222-29.

36 Kreimer AR, Clifford GM, Boyle P, Franceschi S. Human papillomavirus types in head and neck squamous cell carcinomas worldwide: a systematic review. Cancer Epidemiol Biomarkers Prev 2005; 14: 467-75.

37 Lewis JS Jr, Chernock RD, Ma XJ, et al. Partial p16 staining in oropharyngeal squamous cell carcinoma: extent and pattern correlate with human papillomavirus RNA status. Mod Pathol 2012; 25: $1212-20$

38 Thomas J, Primeaux T. Is p16 immunohistochemistry a more costeffective method for identification of human papilloma virusassociated head and neck squamous cell carcinoma? Ann Diagn Pathol 2012; 16: 91-99.

39 Begum S, Cao D, Gillison M, Zahurak M, Westra WH. Tissue distribution of human papillomavirus 16 DNA integration in patients with tonsillar carcinoma. Clin Cancer Res 2005; 11: 5694-99.

40 Hafkamp HC, Speel EJ, Haesevoets A, et al. A subset of head and neck squamous cell carcinomas exhibits integration of HPV 16/18 DNA and overexpression of p16INK4A and p53 in the absence of mutations in p53 exons 5-8. Int J Cancer 2003; 107: 394-400.

41 Reimers N, Kasper HU, Weissenborn SJ, et al. Combined analysis of HPV-DNA, p16 and EGFR expression to predict prognosis in oropharyngeal cancer. Int J Cancer 2007; 120: 1731-38.

42 Lassen P, Eriksen JG, Hamilton-Dutoit S, Tramm T, Alsner J, Overgaard J. Effect of HPV-associated p16INK4A expression on response to radiotherapy and survival in squamous cell carcinoma of the head and neck. J Clin Oncol 2009; 27: 1992-98.

43 Weinberger PM, Yu Z, Haffty BG, et al. Molecular classification identifies a subset of human papillomavirus--associated oropharyngeal cancers with favorable prognosis. J Clin Oncol 2006; 24: 736-47.

44 Wiest T, Schwarz E, Enders C, Flechtenmacher C, Bosch FX Involvement of intact HPV16 E6/E7 gene expression in head and neck cancers with unaltered p53 status and perturbed pRb cell cycle control. Oncogene 2002; 21: 1510-17.

45 Psyrri A, Licitra L, De Blas B, Celik I, Vermorken JB. Safety and efficacy of cisplatin plus 5-FU and cetuximab in HPV-positive and HPV-negative recurrent and/or metastatic squamous cell carcinoma of the head and neck (R/M SCCHN): analysis of the phase III EXTREME trial. Ann Oncol 2012; 23 (suppl 9): 10180 (abstr).

46 Machiels JP, Subramanian S, Ruzsa A, et al. Zalutumumab plus best supportive care versus best supportive care alone in patients with recurrent or metastatic squamous-cell carcinoma of the head and neck after failure of platinum-based chemotherapy: an open-label, randomised phase 3 trial. Lancet Oncol 2011; 12: 333-43.

47 Van Cutsem E, Peeters M, Siena S, et al. Open-label phase III trial of panitumumab plus best supportive care compared with best supportive care alone in patients with chemotherapy-refractory metastatic colorectal cancer. J Clin Oncol 2007; 25: 1658-64.

48 Peeters M, Price TJ, Cervantes A, et al. Randomized phase III study of panitumumab with fluorouracil, leucovorin, and irinotecan (FOLFIRI) compared with FOLFIRI alone as second-line treatment in patients with metastatic colorectal cancer. J Clin Oncol 2010; 28: $4706-13$.

49 Douillard JY, Siena S, Cassidy J, et al. Randomized, phase III trial of panitumumab with infusional fluorouracil, leucovorin, and oxaliplatin (FOLFOX4) versus FOLFOX4 alone as first-line treatment in patients with previously untreated metastatic colorectal cancer: the PRIME study. J Clin Oncol 2010; 28: 4697-705. 